\title{
A Collaborative Virtual Reality Oil \& Gas Workflow
}

\author{
Ismael H. F. dos Santos ${ }^{1}$, Luciano P. Soares ${ }^{2}$, Felipe Carvalho ${ }^{2}$ and Alberto Raposo ${ }^{2}$ \\ ${ }^{1}$ CENPES - Petrobras Research Center / Petrobras (Petróleo Brasileiro) \\ ${ }^{2}$ Tecgraf (Computer Graphics Group) / Dept. of Informatics / PUC-Rio (Pontifical Catholic University of Rio de Janeiro)
}

\begin{abstract}
The current way of designing industrial plants relies on the communication among experts in the field, and on tools that allow the simulation of the site. Virtual reality (VR) tools are used to visualize and interact with complex 3D environments in real time, and several engineering simulations employ VR to foresee the results of complex industrial operations. The research project described here presents a Service Oriented Architecture aimed to create a collaborative environment, called CEE (Collaborative Engineering Environment) that integrates VR techniques into a system where the execution of different sequences of engineering simulations is modeled as scientific workflows. The focus of this research is on the oil \& gas industry, particularly offshore engineering, where the project of a new production unit is a lengthy, expensive and usually is conducted by different specialists who are geographically distributed. Among the integrated engineering simulations are those involving structural calculus, hydrodynamics, naval engineering with mooring systems, meteo-oceanography, and others. The main objective is to improve the users' interpretation capacity and skills while providing visualization tools for a better understanding of the results.
\end{abstract}

Index Terms - collaborative virtual environments, offshore engineering, three-dimensional graphics, virtual reality.

\section{INTRODUCTION}

Oil \& gas industry has seen increasing costs for finding and lifting hydrocarbons, especially in remote locations, ultra-deep water reservoirs or hostile environments. The development of deep-water oil \& gas reserves constantly faces the challenge of trying to reduce the costs of all components and activities. Therefore, high performance computing, visualization and remote collaboration technologies are being extensively used to improve productivity, leading to better cost-effectiveness.

Earth sciences and engineering have to manage and interpret increasing amounts of data captured from the environment or generated by computer simulations. The typical work of scientists and engineers consists in detecting features, measuring them, and finally generating a model that attempts to explain those observed features. This visual approach to science and engineering is powerful, as the human brain excels at identifying patterns visually.

Manuscript Received on October 1, 2011.

E-mail: \{ismael,lpsoares,kamel,abraposo\} @ tecgraf.puc-rio.br
As Edward Tufte [1] wrote almost three decades ago, "At their best, graphics are instruments for reasoning about quantitative information. Often the most effective way to describe, explore and summarize a set of numbers - even a very large set - is to look at pictures of those numbers".

Visualization and remote collaboration technologies help us to bridge the cost-effectiveness problem. In the past years, the industries have achieved sensitive gains in efficiency and effectiveness when carrying out projects using virtual reality technologies. Oil companies were among the first to make industrial use of the so-called virtual reality centers, equipped with immersive projection systems with large display walls and videoconference tools, among other solutions. Three-dimensional geometric modeling, scientific visualization and immersive virtual environments, commonly used in these facilities, pushed the limits of teamwork activities in geosciences and engineering.

VR visualization technologies enhance the content knowledge within any engineering design activity. When used in conjunction with collaboration, VR visualization provides valuable insights for better decision support with risk mitigation. Dodd [2] has mentioned that the next big management push is the empowerment of interdisciplinary teams with collaboration tools that include remote and immersive visualization on the desktop. With this in mind, we emphasize that the combination of collaborative tools and VR visualization constitutes a powerful asset in any software solution for large-scale engineering projects (LSEP).

The concept of Problem Solving Environment (PSE) promises to provide large scale engineering projects with integrated environments for problem solving specialized in the application domain, increasing team members' productivity allowing them to focus on the problem at hand rather than on general computational issues. A PSE is a specialized software system that provides all the computational facilities needed to solve a target class of problems [3]. PSEs allow users to define and modify problems, choose solution strategies, interact with and manage appropriate hardware and software resources, visualize and analyze results, record and coordinate extended problem solving tasks.

Collaborative Problem Solving Environments (CPSE) focus on the development of a PSE coupled with collaborative environments to support the modeling and simulation of complex scientific and engineering problems. For large-scale engineering projects, a CPSE should focus on the development and integration of scientific tools and technologies, coupled 
with visualization capabilities and collaborative environments to support the modeling and simulation of complex scientific and engineering problems in a collaborative way. Such capabilities enable engineers to easily setup computations in an integrated environment that supports the storage, retrieval, and analysis of the rapidly growing volumes of data produced by computational studies.

CEE (Collaborative Engineering Environment), our proposed solution, was conceived as a CPSE especially tailored for assisting the control and execution of shared engineering projects involving geographically distributed teams [4]. It should also allow an easy integration of different engineering applications providing team workers with means of information exchange, aiming to reduce the barriers imposed by applications with limited or no collaboration support.

The development of CEE is motivated by the necessity of developing effective solutions for collaboration among team members during the execution of large and complex offshore engineering projects. Such projects usually require the execution of a number of engineering simulations, encapsulated as engineering services. These are combined in different orders and rearranged in different subsets according to project requirements, comprising different scientific workflows [5] especially tailored for the offshore engineering field.

By means of a scientific workflow management system (ScWfMS), users are able to orchestrate the execution of engineering simulations as workflow tasks that can be arranged in many different ways. Within a workflow, as its last step, the most interesting cases can also be selected for visualization in an immersive collaborative session. A simplified grid-computing infrastructure seamlessly integrated into the system supports the distributed execution of simulations. Collaboration support is provided by a videoconference system [6], a mechanism for creating private and shared 3D annotations connected to engineering artifacts, and additional visualization feed-through mechanisms that improve system awareness. Those features compose a collaborative problem-solving environment that enables engineers to set up computations in an integrated environment [3]. CEE is therefore composed of the following tools for distributed group work:

1) Virtual reality visualization tool especially tailored for collaborative visualization of engineering simulations in an immersive or desktop environment [32]t;

2) Scientific workflow management system based on BPEL (Business Process Execution Language) [7]workflows, used as a process-oriented tool to control simulations;

3) Videoconference system developed to support human communication, providing integrated audio and video channels, subject to defined control policies [6].

In the following sections we present some aspects of the developed system. In section 2 we outline the main characteristics of offshore engineering projects, discussing some of the problems addressed. Related works that inspired the development of the system are presented in section 3. In section 4 , we describe the main architecture of the CEE. Some application scenarios are presented in section 5, and conclusions in section 6 finish the paper.

\section{OFFSHORE ENGINEERING PROJECTS}

In the offshore engineering field, the project of deep-water production systems, including oil platforms or ships and all equipment that plays a part in the production process, is currently designed by means of complex computer modeling systems. The design of a new production unit is a lengthy and expensive process, which can last many years and consume hundreds of millions of dollars, depending on the complexity of the unit and the maturity of the technology required to make the project technically and economically feasible.

Offshore engineering projects involve not only geographically distributed teams but also teams of specialists in different areas using different software tools, both commercial and internally developed. While the interoperability of those tools is still an issue, it is a mandatory requirement for any collaborative solution.

Due to their huge complexity, offshore engineering projects are divided into smaller interrelated subprojects, each one dealing with an abstract representation of the others. Because decisions are interdependent, collaboration is a key issue in this area. Each team activity or decision can affect others. For example, during the design of an oil platform, changing the position of large and heavy equipment in the process plant can compromise the stability of the production unit. In some cases there is also an intrinsic coupling among the solutions of the different subprojects, which requires intensive interaction and discussion among the teams involved. This is the case of the mooring system and of the production riser subsystems (Fig. 1). On the one hand, if the mooring system allows the production unit to experience high fluctuations, this can damage the production risers; on the other hand, the mere presence of risers helps to reduce the movements of the production unit, contributing positively to the equilibrium of the system. In order to achieve collaboration and interoperability between those subprojects, a software-based interface is required.

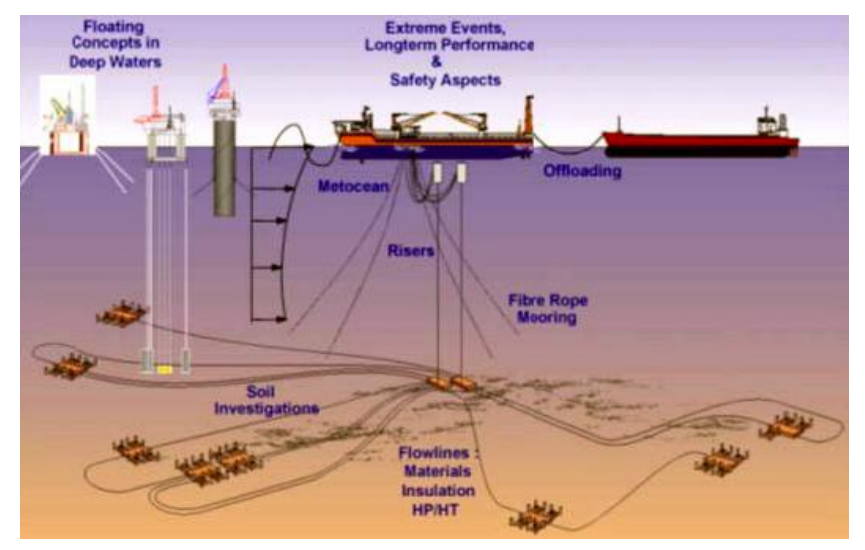

Fig. 1. Deep-water production system layout (Color Plate 1)

A different problem, related to the need to represent multiple data, is that although specialists deal with the same information (platforms, risers, mooring systems, etc.) they usually have 
different data representations for those objects according to the needs of each activity. This requires support for multi-resolution representation of the data. For example, in structural and naval engineering the models usually have dense polygonal meshes, with a few objects representing the outline of the artifacts, suitable for numerical analyses of static and dynamic stability studies. The need for different data representation imposes one of two alternatives: either support for multi-resolution representation is implemented, or rendering performance is improved in order to deal with data.

Another challenge posed by offshore engineering projects is related to the visualization of large engineering simulations. During the conceptual design phase of an industrial plant, several simulations must be carried out to assess the robustness and feasibility of the project. Some of these simulations may require huge computational effort to be processed, such as through the use of computer clusters. Visualization should be as precise as possible in order to provide the user a full understanding of the results.

$\mathrm{CAD} / \mathrm{CAE}$ models usually have objects with coarse grid meshes suitable for good visual representation, but the problem is that all the objects that comprise the artifact are represented, yielding huge models. Another aspect is that CAD/CAE models are the best representatives for real artifacts, so there is a tendency to use those models directly in simulations and visualizations. Even today, realistic real-time visualization of those models is a very complex problem in computer graphics [8].

This research project focuses on the development and integration of scientific and visualization tools and technologies coupled with collaborative environments that support the modeling and simulation of complex engineering projects. CEE is designed to solve simple or complex problems, providing support for both rapid prototyping and detailed analysis.

In conclusion, according to the above challenges presented for Offshore Engineering projects, CEE was conceived as a useful solution for better controlling and executing specialized engineering projects through the use of its collaboration and visualization capabilities.

There are other important problems that can be tackled with CEE such as: training and security simulations; design, planning and optimization of marine installations and sub-sea layout arrangement of production systems; remote operation and interventions on subseasystems; preparing maintenance and inspections plans in production units; planning oil pipeline installation and monitoring and emergency scenario applications. In this paper, in section 5, we present the use of CEE for controlling and executing structural analysis projects of riser and mooring systems.

\section{RELATED WORK}

In this section we present a few relevant works that motivated this research towards the construction of a collaborative immersive visualization environment for visualizing engineering simulations.

Dynamic data-driven approaches, such as the Data Driven
Multiphysics Simulation Framework (DDMSF), are increasingly becoming more feasible because of the confluence of several technologies. First, advanced sensor technologies have improved the ability to capture data faster and at higher resolution. Second, Grid Computing infrastructure aims to dynamically and seamlessly link powerful and remote resources to support the execution of large scale and disparate processes characterizing a particular problem. Among all DDMSF components, the Discover Computational Collaboratory [9] strongly inspired our proposed solution. Its overall objective is to realize a CPSE that enables geographically distributed scientists and engineers to collaboratively monitor, interact with, and control high performance applications in a truly pervasive manner, transforming high-performance simulations into modalities for research and instruction.

Paventhan et al. [10] proposed the creation of WindTunnel, a scientific workflow for wind tunnel applications. They observed that scientific and engineering experiments often produce large volumes of data that ideally should be processed and visualized in near real time. The difficulty to achieve this goal is that the overall turnaround time from data acquisition, transference to a data processor, and visualization of the results is frequently hindered by factors such as manual data movement, system interoperability issues, manual resource discovery for job scheduling, and disparate physical locality between the experiment and the user workstation. The authors argue that customized application-specific workflows could reduce the time taken to accomplish a job by automating dataflow-driven activities, supplementing or replacing manual user-driven tasks. WindTunnel provides a series of workflow activities that allow users to compose sequential workflows and seamlessly access grid services. WindTunnel approach also inspired the development of the proposed system by combining the Scientific Workflow Management System (ScWfMS) with the execution of engineering applications in a Grid infrastructure.

Vistrails [11] is a visualization management system that provides a scientific workflow infrastructure, which can be combined with existing visualization systems and libraries. A key feature that sets Vistrails apart from previous visualization systems is support to data exploration. It separates the notion of dataflow specification from its instances. A dataflow instance is a sequence of operations used to generate a specific visualization. The approach adopted in Vistrails inspired the strategy behind the software developed here. Among the differences implemented at CEE is the use of a BPEL (Business Process Execution Language) in the ScWfMS [12].

Parker et al. [13] describe SCIRun, a problem-solving environment (PSE) that allows users to interactively compose, execute, and control a large-scale computer simulation by visually "steering" a dataflow network model. SCIRun supports parallel computing and output visualization, but originally has no mechanisms for experiment managing and archiving, optimization, real-time collaboration, or modifying the simulation models themselves. Paraview [14] is a kind of PSE for visualization that allows the interactive creation and manipulation of complex visualizations. Paraview is based on the notion of dataflow, and provides visual interfaces to produce visualizations by assembling pipelines out of modules 
that are connected in a network.

However, both SCIRun and Paraview have significant limitations. First, there is no separation between the definition of a dataflow and its instances. In order to execute a given dataflow with different parameters, users need to set these parameters manually through a GUI - not scaling to more than a few visualizations. Second, modifications to parameters or to the definition of a dataflow are destructive. Despite their limitations, SCIRun and Paraview show the importance of combining visualization with PSEs.

In the Geology field, Kreylos et al. [15] presented an approach for turning immersive visualization software into scientific tool. They created immersive visualization measurement and analysis tools that allow scientists to use real word skills and methods inside Virtual Environments. They have also conducted some informal studies to determine the impact of using VR methods on some geosciences tasks such as Geological Mapping and Displacement Analysis. As shown by GMDA, the usage of a VR Visualization system to debug engineering simulations is a very powerful tool for LSEP. Their observation that VR visualization enabled scientists to make more accurate observations in less time and with more confidence has also motivated to include a VR Visualization system as an important component of the CEE architecture.

Service Oriented Architecture (SOA) [16] provides a platform for building application services with the following characteristics: loose coupling, location transparency and protocol independence. An SOA application that influenced this research is the Integrated Asset Management framework (IAM). IAM provides to its users a front-end modeling environment for specifying and executing a variety of workflows from reservoir simulations to economic evaluation [17]. The IAM framework is intended to facilitate seamless interaction of diverse and independently developed applications that accomplish various sub-tasks in the overall workflow.

In Fig. 2 we present a comparison of the features provided by CEE and the features presented by the solutions mentioned above. It can be seen that CEE has a wider spectra addressing the most important requirements of LSEPs.

\begin{tabular}{|c|c|c|c|c|c|c|}
\hline & CEE & DDMSF & $\begin{array}{l}\text { Wind } \\
\text { Tunnel }\end{array}$ & Vistrails & GMDA & IAM \\
\hline \multicolumn{7}{|l|}{ ScWfMS } \\
\hline Scripting Language & $\alpha$ & $x$ & $\checkmark$ & 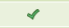 & - & $ه$ \\
\hline Visual Tool for Composition & 8 & $x$ & $ه$ & $\checkmark$ & - & 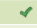 \\
\hline Data Provenance & 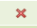 & $x$ & $x$ & 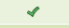 & - & 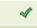 \\
\hline \multicolumn{7}{|l|}{ Grid Computing Infrastructure } \\
\hline Job Submission & 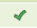 & $\checkmark$ & $\$$ & $\checkmark$ & - & 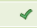 \\
\hline Job Monitoring & 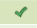 & 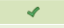 & $ه$ & 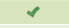 & - & 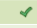 \\
\hline \multicolumn{7}{|l|}{ Collaboration } \\
\hline Collaborative Portal & 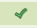 & $\checkmark$ & × & $x$ & - & * \\
\hline Videoconference & 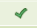 & $x$ & $x$ & $x$ & - & $\times$ \\
\hline \multicolumn{7}{|l|}{ Visualization } \\
\hline Virtual Reality & 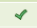 & $x$ & - & - & $\$$ & - \\
\hline Collaborative Visualization & 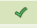 & $x$ & x & $x$ & - & - \\
\hline $\begin{array}{l}\text { Visualization Tools } \\
\text { (3D Annotations, Measurements, Virtual Tours) }\end{array}$ & $\alpha$ & $x$ & - & - & $a$ & - \\
\hline Scientific Visual ization & 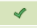 & $\checkmark$ & - & 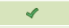 & $\checkmark$ & - \\
\hline Computational Steering & x & $x$ & - & 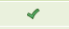 & $x$ & - \\
\hline \multicolumn{7}{|l|}{ Data Access Service } \\
\hline Data stage In/out & $x$ & $\checkmark$ & $\checkmark$ & $\checkmark$ & $x$ & $ه$ \\
\hline Querying and Retrieval Mechanisms & $x$ & $\checkmark$ & $\checkmark$ & $x$ & $x$ & $x$ \\
\hline & & & & $\begin{array}{l}x \\
z \\
-\end{array}$ & $\begin{array}{l}\text { Does not } \\
\text { Provide } \\
\text { Not Appli }\end{array}$ & \\
\hline
\end{tabular}

Fig. 2. Comparison between CEE and related collaborative solutions.

In the upstream segment of the oil \& gas industry, the determination of optimal well path trajectories is a challenging problem for reservoir engineers [18]. Gruchalla et al. [19]investigated the benefits of immersive VR for well-path editing. They reported higher speed and accuracy in immersive systems than in a desktop system, based on a study with 16 participants who planned the paths of four oil wells. Each participant planned two well paths on a desktop workstation with a stereoscopic display, and two well paths in a CAVE-like immersive virtual environment [20]. Fifteen of the participants completed the well-path editing tasks faster in the CAVE environment than in the desktop environment. The higher speed was accompanied by a statistically significant increase in correct solutions. The results suggest that an immersive environment allows for faster and more accurate problem solving in a complex interactive three dimensional domain.

The Fraunhofer Gesellschaft VRGeo Consortium [21] is an oil \& gas international consortium for the development of visualization technology for geoscience and engineering applications in virtual environments. VRGeo has presented many significant contributions on the use of VR technology, especially in the area of collaborative work in virtual environments. Simon et al. [22] presented a qualitative and quantitative study comparing usability and interaction performance with multi-viewpoint images, where a large-screen projection-based stereoscopic-display system is shared by a small group of people, each with their own viewpoint.

Another work was the VRGeo Demonstrator Project, for the interactive analysis of complex geological surfaces and volumes in an immersive VR system through co-located collaboration [23]. The authors present a new interaction paradigm that allows multiple users to share a virtual space in a conventional single-view stereoscopic projection-based display system, with each user manipulating the same interface and having a full first-person experience in the environment.

The effective integration of "smart" graphical user interfaces, with some advisory support, Scientific Visualization, Virtual Reality techniques, Engineering Analysis and Modeling Tools aid in the automation of modeling analysis and data management for large and complex engineering projects. To enhance engineers' ability to share information and resources with colleagues at remote locations, collaborative and real-time technologies integrated into CEE provided a unified approach to the scientific and engineering discovery and analysis process.

A combination of a collaborative problem-solving environment and VR visualization, which is not addressed by the aforementioned visualization systems, constitutes a strategic enabler for successful data exploration and knowledge dissemination among workers in engineering enterprises. VR visualization technologies enhance content knowledge within the engineering disciplines. In conjunction with collaboration, both provide valuable insights for better decision support with risk mitigation.

\section{CEE ARCHITECTURE}

In order to achieve its goals, the CEE architecture is a 
composition of different computer-supported collaborative work technologies. The system is composed of a Collaborative Visualization Environment (CVE) based on a Virtual Reality Visualization (VRV) tool and a Videoconference system (VCS); a Scientific Workflow Environment (ScWfE) with an associated Grid Computing Infrastructure (GCI) for executing large engineering simulations; and a Project Management Environment (PME) responsible for controlling the overall execution of the project and keeping track of all the information and different artifacts generated during the whole lifecycle of the project.

The CEE allows users to solve their problems collaboratively, using predefined scientific workflows or assembling new ones as necessary. Each workflow comprises a sequence of simulations, usually ending with a collaborative visualization supported by a VRV tool.

To achieve this, CEE was devised as an extensible, flexible and system-independent platform, allowing a transparent flow of information among different levels, systems and models. The challenges for building an effective and useful solution can be scrutinized according to the following domains:

1) Collaborative VisualizationEnvironment- this domain encompasses very different challenges from the areas of CSCW and Virtual Reality.

a) Collaborative Work - in this domain there is the necessity of providing effective human-to-human interaction and communication for solving conflicts and enhancing group productivity. Also there is the need of some support for coordinating the execution of tasks.

b) Virtual Reality Visualization - high performance and scalability are important aspects of virtual environment architectures intended to support execution of large shared virtual worlds over long periods of time.

2) Scientific Workflow Environment - this domain includes challenges related to the control of the execution of engineering simulations

a) InteroperabilityandDistributed Execution - in this domain there is a myriad of software that specialists, potentially geographically distributed and using distributed resources, are forced to use in order to accomplish their tasks in a reasonable time. This requires the solution to have the ability to be easily and seamlessly distributed and demands a high level interoperability among its components.

b) Data provenance - Data provenance is the capacity of maintaining information of how a given data product was generated [24] and has many uses, from purely informational to enabling the representation of the data product. It is a very important feature for any CPSE once scientists and engineers often create several variations of a workflow in a trial-and-error process when solving a particular problem.

3) Project ManagementEnvironment - this domain points to the necessity of keeping track of all the documents and artifacts generated during project's life-cycle. Multiple and different visions of the on-going project must be provided while users have different background (e.g. managers, engineers) and need different types of information to accomplish their duties.

The CEE conceptual model, presented in Fig. 3, handles the above-mentioned challenges by creating specific services for them, constituting a service-oriented architecture (SOA) solution [4].

The CVE is responsible for managing user interaction. The VCS and the VRV services work closely coupled with the Collaboration Manager Service (CMS) to enable the creation of collaborative visualization sessions.

The ScWfE was created to help users build engineering workflows and seamlessly execute them in a GCI. For interoperability among applications, a common format for data exchange among engineering applications was designed and implemented [30]. More generally, to support distributed execution, we use the interoperability characteristics of the ScWfMS and the distributed execution support provided both by the GCI and also the support provided by the SOA backbone infrastructure furnished by the Enterprise Service Bus (ESB)[25].

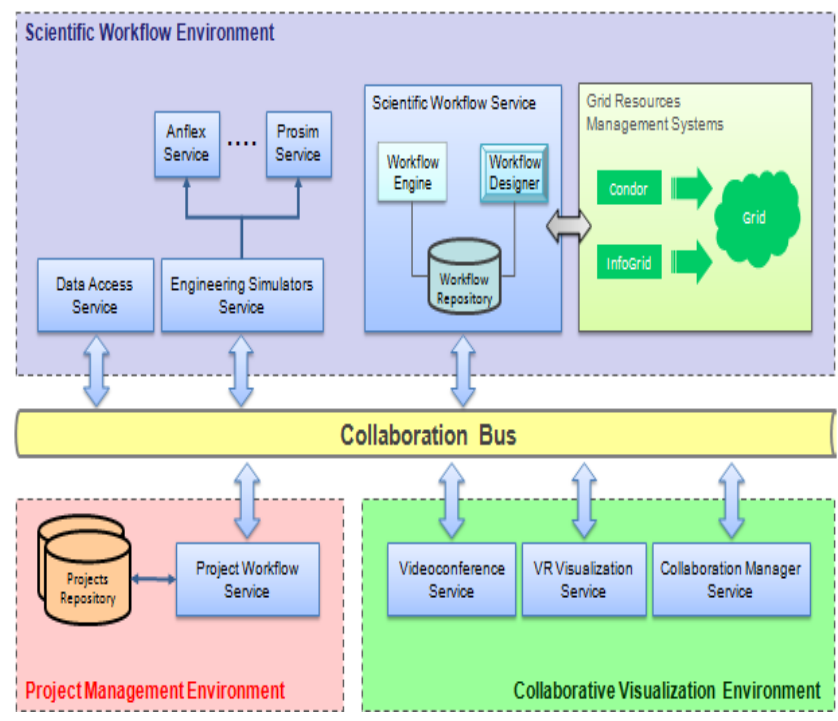

Fig. 3. CEE conceptual model.

The Engineering Simulations service provides web service interfaces [26]for the remote execution of engineering simulations. Those simulators are, among others, Anflex [27], a finite-element riser analysis software, and Situa/Prosim [35], a finite-elementcoupled analysis software for floating production systems design.

In the following sections we proceed discussing further details of the CEE conceptual model main components.

\subsection{Scientific Workflow}

Ellis et al. [28]implement workflow management systems (WfMSs) as tools to assist in the specification, modeling, and enactment of structured business processes within organizations. These systems are a special type of collaboration technology, which can be described as "organizationally aware groupware". 
Although the above definitions make reference to "business processes", WfMSs are not only employed by business applications. Weske et al. [29] identified that, in a scientific environment, scientists will typically specify their workflows themselves, while, in a business environment, a system administrator is commonly responsible for this task. Another characteristic of scientific workflowspointed in their work is the need to trace workflow executions. An engineer may need to reuse a workflow in order to reproduce results. The operations a user performs on a given data must be recorded in order to provide engineers with the benefits of successful and unsuccessful workflows furnishing provenance information for the executed workflow [24].

Scientific workflows often begin as research workflows and end up as production workflows. Early in their lifecycle, they require considerable human intervention and collaboration; later they begin to be executed more automatically. Thus, in the production mode, there is typically less room for collaboration at the scientific level, and the computations are more long-lived. During the research phase, scientific workflows need to be enacted and animated (fake enactment) far more intensively than business workflows. In this phase, which is more extensive than its counterpart in business workflows, the emphasis is on execution with a view to design, and thus naturally includes iterative execution. The corresponding activity can be understood as "business process engineering" (BPE), a kind of an engineering counterpart to the Business Process Management (BPM). For this reason, the approaches for constructing, managing, and coordinating process models are useful also in scientific settings. In this way, Scientific Workflows are to Problem Solving Environments what Business Workflows are to Enterprise Integration (EI).

Scientific Workflow Management Systems (ScWfMS) are more data-flow oriented while Business Workflow Management Systems (BWfMS) are more control-flow oriented. BWfMS require the coordination of a number of small messages and document exchanges. In ScWfMS usually no documents undergo modifications. Instead, often a dataset is obtained via analysis and transformation of another dataset. BWfMS need complex control flow, but they are not data-intensive pipelines. On the other hand, ScWfMS must deal with the heterogeneity, complexity, volume, and physical distribution of scientific data. In addition to these data problems, ScWfMS often deal with legacy or third-party programs, which can also be heterogeneous, and possibly with no source code available.

In a typical scenario, data is usually passed from one program to another in order to complete several steps of the simulation. Once in the CEE the sequence of operations to perform an engineering simulation are modeled as scientific workflows, there is an interoperability problem, since in most of the cases, data conversion steps are needed every time a different program needs to be run over the data. To solve the data interoperability problem, allowing applications to share engineering data in the context of such scientific workflows, a unified data format called GXML have been designed and implemented [30]. As the name says, it is based on XML, which can be easily handled by applications using standard XML APIs.

For scalability purposes GXML classifies data as light and heavy, according to the amount of information it represents. In this sense, light data is allowed to be stored in the GXML file's body, while heavy data is stored in HDF5 (Hierarchical Data Format) [32] in an internally compressed format and described in the GXML file. HDF5 provides efficient ways for reading and writing huge volume of data which is very important for engineering and scientific data.

\subsection{Support for Collaborative Work}

Collaborative systems should allow multiple users not only to interact with shared objects but also to communicate and coordinate their actions. Collaboration may be seen as the combination of communication, coordination and cooperation [31]. Communication is related to the exchange of messages and information among people. Coordination is related to the management of people, the interdependencies among their activities and the resources used. Cooperation is the production of common artifacts taking place in a shared space through the operations available to the group.

In our environment, support for collaborative work is provided by a ScWfMS, a VCS and a Collaboration Bus (CBus), a collaborative infrastructure support for integrating the execution of engineering applicationswith the VRV component allowing the users to collaboratively visualize their results and optionally create virtual Annotations, to share knowledge about the engineering artifacts being visualized..

The ability to collaboratively create persistent annotation on the model improves its usefulness. An annotation, in our context, is any information (text, image, video or hyperlink) that users want to add to their projects to enrich the content or just for documentation, having a private or public (shared) scope. Annotations can be associated to any artifact manipulated during a collaborative visualization session, which usually happens at the end of the execution of any sequence of engineering simulations.

An annotation may represent instructional information related to a sequence of operations that should be undertaken during an equipment maintenance intervention, for instance. It can also be any textual information used to highlight interesting or anomalous events observed in the simulation results. Examples of such events could be unexpected values for an engineering figure, violations of integrity, etc. Annotations can also have a more dynamic behavior, for instance representing the distance between different objects that should be monitored during a simulation, like the distance between two different elements in two different ascending risers to a floating production system.

Cooperation also occurs when assembling engineering workflows that will be used to orchestrate the execution of engineering applications, and also during the visualization of results, when users can collaborate to better understand the model. Users can also share persistent annotations about interesting facts, as previously discussed.

There are different types of awareness support provided in CEE. The most important scenarios for awareness support are:

1) Event monitoring - observing what is going on in the $\mathrm{VRV}$, in all separate parts, and providing active notification to the right person, at the right time and in 
the selected subsystem;

2) Workspace awareness - providing control of collaborative interaction and changes in the user location;

3) Mutual awareness - allowing users see each other's identity and observe each other's actions;

4) Group awareness - facilitating the perception of groups of interest by connecting people who need to collaborate more intensely. Group awareness enables the user to build his own work context and to coordinate his activities with those of others'. Informal communication provided by a Videoconference system enhances team awareness.

User awareness is a very important subject for CEE once it is a desirable feature to provide mutual awareness during the collaborative visualization session allowing users see each other's identity and observe each other's actions specially when creating Annotations in the model.

The Collaboration Manager service (CMS) is responsible for managing user participation in a collaborative session and for integrating the resources of Virtual Reality Visualization and Videoconference system. There are three kinds of sessions available:

1) Informal - where each participant uses its individual telepointers all the time. There is no mediation of camera movements and the users are free to move around the scene propagating the camera movements to others. In this model, once a collaborative session is created, audio and video can be used at any time by all users. The only mediation mechanism supported is furnished by the social protocol available whenever a videoconference is started.

2) Classroom - where one specific participant, the instructor, acts as a coordinator of the session which means that all camera movements he performs are followed by other users, while the other participants have their telepointers disabled. The instructor also controls the audio and video channels of the participants, and he is also allowed to pass control of collaboration resources (telepointers, camera control, etc.) among participants, taking it back at any time.

Users can request the coordination role to the current coordinator, who can accept or reject the request, generating a visual feedback to the requestor. Upon the occurrence of a "change coordinator" event in a CEE collaborative session, all users are notified by the awareness mechanism.

3) Lecture - where one specific participant, the speaker, acts as a coordinator of the session, with the same characteristics as in the Classroom session. In this type of session there is no exchange control between the coordinator and participants and the participants can only receive audio and video stream from the coordinator. This model of session is used for example when the speaker wants to create a virtual guided tour showing important details of the results of a simulation to other participants.

For each kind of session, users have their status determined according to their role (coordinator or participant). In an
Informal collaborative session users are in a SendAndReceive state where the users are free to move around the scene propagating the camera movements to others.

In a Classroom session, the coordinator is in a SendOnly state where he can only send camera movements and must ignore any camera movements from the other users, All the other participants are in ReceiveOnly state where they can only receive commands and cannot send any camera movements.

The difference between Classroom and Lecture is that in a Classroom session it is possible to request the coordinator role; while in a Lecture session this possibility is forbidden.

At any time, the user can disconnect from the session to do private work, and reconnect later (late join). When this happens, the user's state is resynchronized with the state of the session, which is controlled by the CM service.

The Collaboration Bus (CBUS) is a key component of the overall architecture and provides synchronous and asynchronous communication among the CEE components. It is a communication infrastructure based on the JMS Service Provider, the message-oriented middleware used for providing the public/subscribe and point-to-point paradigms, and the Enterprise Service Bus.

The CEE Awareness service provides appropriate actuators for events received from the CBus. It is responsible for signaling distributed events to the users participating in a collaborative session. On one side, all components trigger events to this CBus, and on the other side awareness components listen to the bus to gather information about what is happening in the system. When users leave a collaborative session or when there is a change in its state from offline to online and vice-versa, "update user" events are triggered in the CBus, and the CEE awareness mechanism sends messages to the VRV and the VCS services notifying the event. These services then signal those events in their user interfaces, making users aware of what happened. Examples of such kind o signaling used in CEE are: different icons in the GUI for showing the state of the users; windows messages warning the entrance of a new user in the session or the leaving of the user from the session, etc.

\subsection{VR Visualization Tool}

The applicability of VR techniques for 3D geometric CAD models has been restricted to design review, virtual prototyping and marketing purposes, mainly in the automotive and aircraft industries. More recently, 3D CAD models are starting to show their potential in VR applications for diverse purposes, such as ergonomic studies, safety training for Health, Safety Environments (HSE), and visualization of physical simulations, project documentation and real-time operational data.

During the research project described in this paper a tool, called Environ[33]was developed to facilitate the use of massive CAD models in VR applications. It is a system composed of a $3 \mathrm{D}$ environment for real-time visualization and plugins to import models from other applications, allowing users to view and interact with different types of 3D data, such as refineries, oil platforms, risers, pipelines and terrain models. 

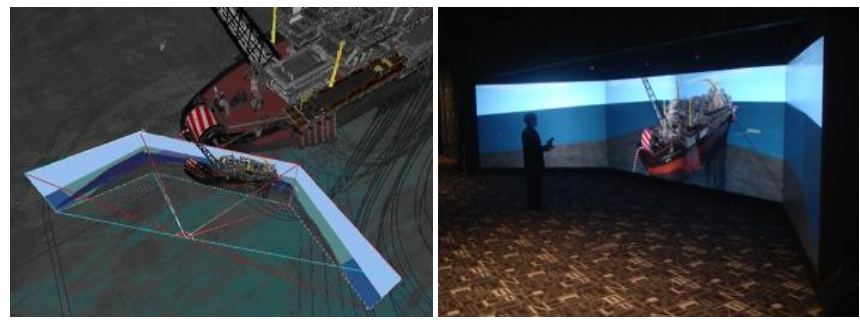

Fig. 4. Virtual representation of a cave and user interaction.

This tool is integrated into the complete system, offering resources for real-time 3D visualization and interaction with CAD models with enough realism and performance to be used for collaborative virtual prototyping, design review, change management, training, and visualization of simulations, among other activities. Fig. 4 shows some users using this tool at one of the Petrobras (the Brazilian government-owned oil company) Research Centre Visualization and Collaboration immersive rooms.
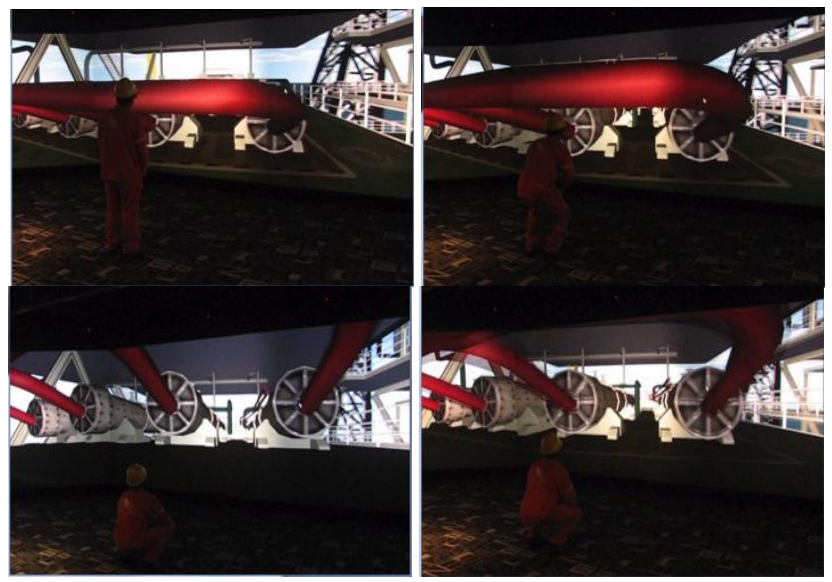

Fig. 5. Head-tracking with dynamic generation of viewpoints(Color Plate 2)

The use of stereoscopy for immersive applications in engineering is essential. This is achieved through user head-tracking, multi-projection calculations and the determination of dynamic parameters of stereoscopy [34]. Head-tracking helps to determine the relative position of the user in the viewing screens. This position is the input to build suitable viewpoints. Fig. 5 shows examples of different views from tracking the user's head.

Finally, in our scenario, the proper determination of the stereoscopy parameters is directly related to the navigation techniques of the immersive environment. For example, the operation "Go To" has as prerequisite the determination of the target object to start the navigation. Based on the size of this object, the representation is scaled and the stereoscopic parameters (eye distance and zero parallax distance) are dynamically calculated. A sequence of images illustrating the different scales used while the user is navigating in the scene on a 3-sided open cave is presented in Fig.6. In the images on the left side the "preview windows" shows the application of the dynamic scale algorithm applied to the model according to the user navigation in the scene.

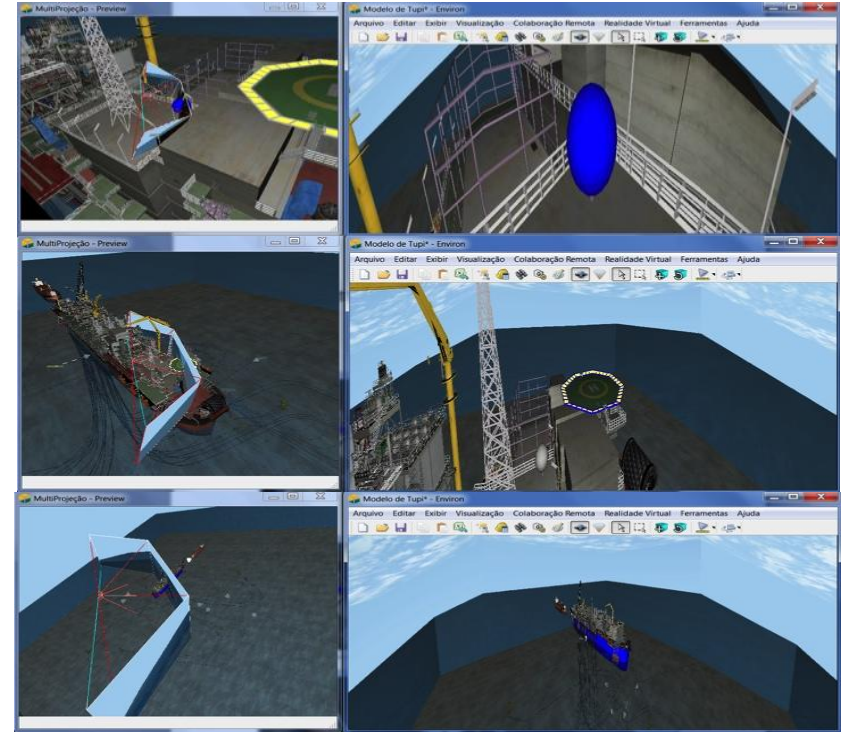

Fig.6. Cave scales based on a target object.

\section{APPLICATION SCENARIOS}

\subsection{Collaborative Riser Analysis Workflow}

An important step in deep-water oil exploitation is the elevation of the oil from depths over one thousand meters from the seabed to the floating production facilities on the surface. Oil platforms use ascending pipes, called risers, which are tubular structures that convey oil and/or gas from the wellhead on the sea floor to the platform's separator system tanks [35]. To certificate the operation of the risers for their entire lifecycle (30 years or more), simulations of the stress applied to the riser system are conducted based on meteo-oceanographic data about wind, tide and water currents. Simulations are made under extreme environment conditions to test stress resistance. It is important to perform fatigue analysis studies to evaluate the most critical regions of the risers affected by cyclical stress in order to guarantee their integrity during their lifetime.

As a result of their complex behavior, the development and application of rigorous structural and fatigue analysis tools for offshore platforms and risers installed by Petrobras has become a relevant task to engineers.

The analysis of risers requires the use of a suite of standalone programs. Since the analysis process is complex and handles a large amount of data files, this simulation is very hard to execute and manage manually. Furthermore, as the output files of one program are the input of another, it is necessary to make countless data manipulation and transformation operations using additional tools or programing new scripts to accomplish these tasks. Not only is this expensive in terms of time, it can also be error prone. In this context the use of a scientific workflow to represent and execute that sequence of simulations is an important feature of the system.

Fig.7 shows the main components of the CEE interaction. Initially after the user is logged in the system, the User Service on the client machine registers the user in the Collaboration Manager Service on the server, all services that the user's machine is able to support (Environ Service, VC Service, etc) is also registered on the CEE server Service Registry. 


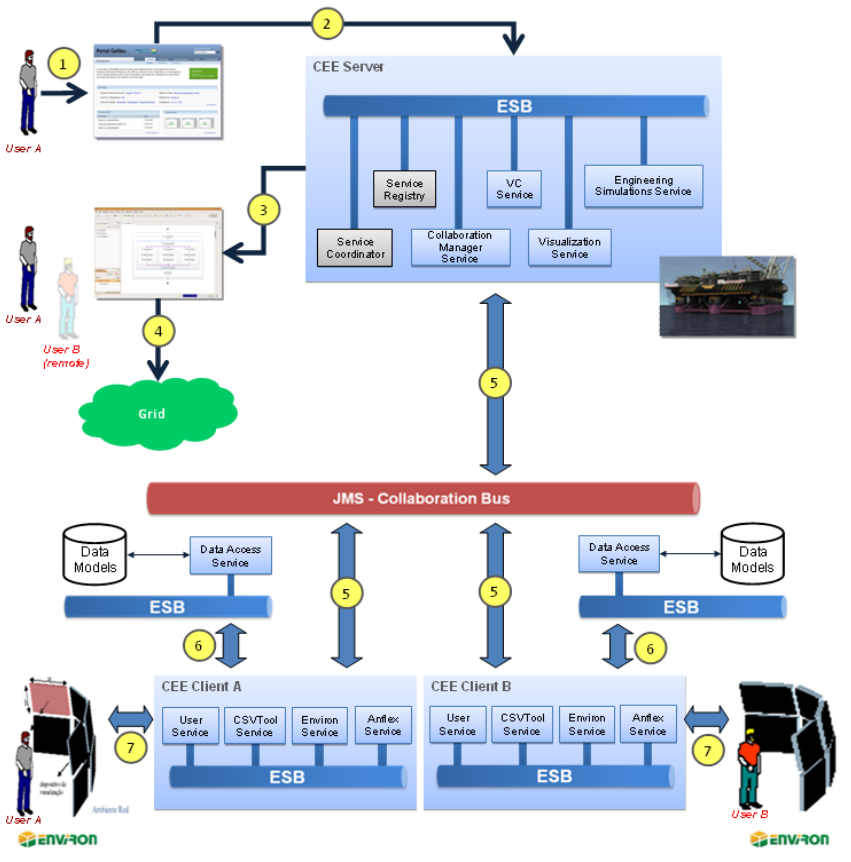

Fig.7. Overview of the user interaction with CEE (Color Plate 3).

After registration of its services on the server, User A accesses the CEE Portal (1 in Fig.7) through a web browser to request the execution services on the CEE server creating a collaborative session on his be-half (2). He can also invite User $\mathrm{B}$ to participate in the session. An example of a collaborative session instance would be when User A models collaboratively with User B a scientific workflow (3). When the model is assembled and all input parameters of the workflow are informed, User A can submit the workflow as a job for executing on a Grid integrated into the CEE infrastructure (4).Usually after the submission of the workflow the users can continue submitting other jobs or can close the session and wait for the completion of its jobs.

Examples of simulations, in the context of Offshore Engineering, can be: design of a mooring system for a production unit or a fatigue analysis of the riser system. Upon finishing its execution, the results of the concrete workflow may be visualized in a Collaborative Visualization Session with User B (5).

During the collaborative visualization session, the users can require the execution of alternative simulations and have its results exhibited automatically (6 and 7).

An Anflex-based BPEL scientific workflow was defined for riser analysis using Anflex simulator. It is controlled by the BPEL engine, which automates the validation process and the certification of riser analysis. The workflow integrates the execution of the Ocean service, the Anflex service and the Grid Job service. In Fig.8 we show the final version of the riser analysis workflow in the BPEL designer.

The workflow starts with an Anflex base case, where the basic configuration is defined as production unit, riser geometry, soil bathymetry and its physical properties, etc. The Anflex service receives user input parameters from the BPEL designer and creates different loading cases according to the different meteo-oceanographic conditions provided by the Ocean service. After that, the BPEL engine instructs the CEE
Grid Job service to submit jobs for executing the simulation program on the available nodes of the associated gridinfrastructure.

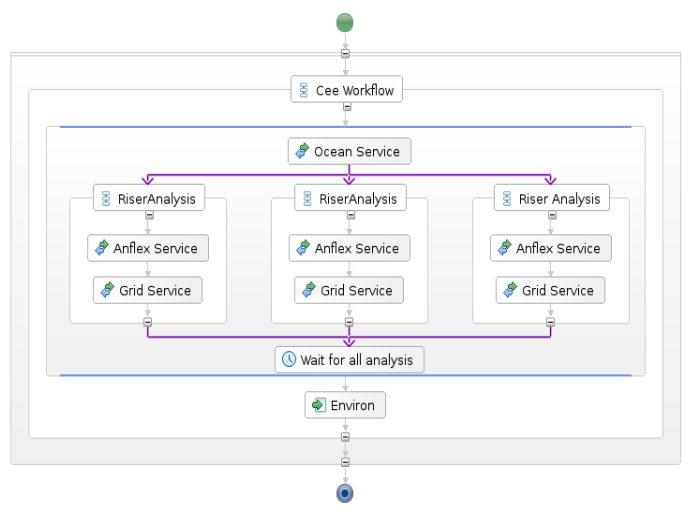

Fig.8. Riser analysis workflow in the BPEL designer.

Fig.9 shows a collaborative visualization session with the presence of two users, represented by two distinct 3D-cursors (avatars), visualizing the simulation results in their desktop with the support of a Videoconference system. Two 3D annotations were created automatically, showing the extreme points of a selected force or strength in the riser. The third annotation was created by the user to register an observation made in this collaborative session.

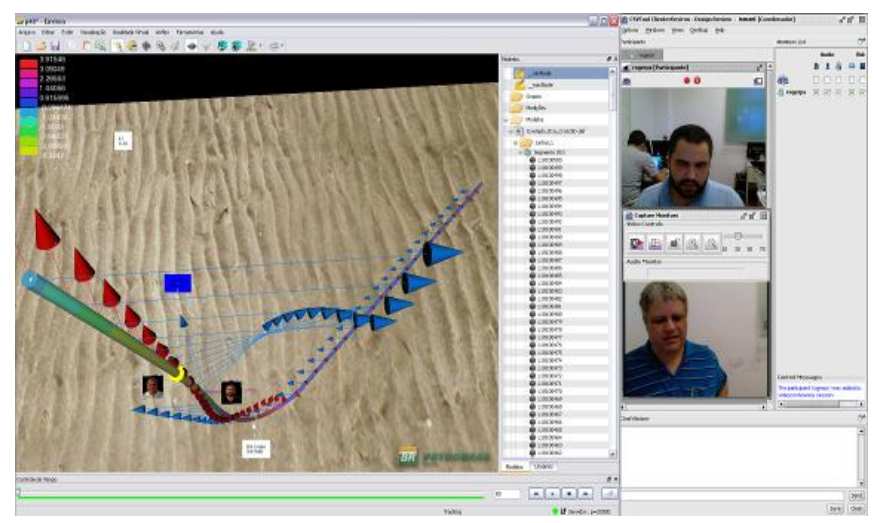

Fig.9. Riser analysis (CAD + videoconference).

The blue arrows represent the water currents that actuate over the riser, while the red arrows represent the direction of the movement of the riser (i.e. instantaneous velocity). Observe that the greater the alignment of those two groups of arrows the greater the influence of the water currents in the final movement of the riser. For that situation we can see that there is no such alignment, which means that other environmental forces (winds and waves) have a greater influence in the final movement of the riser.

In Fig.9, two users (coordinator and a participant) are examining the behavior of a selected element in the riser (green ring), animated through the timeline bar. Note that, the coordinator receives a video stream from the participant, while in Fig.10 the participant receives the image of the coordinator. This way the efficiency of the collaboration is dramatically improved due to the user Awareness obtained by the use of a 
VCS, where the users can see each other and also receive a screen copy of the remote user desktop. Each user is represented by its own avatar associated with the position of its telepointers. The transmission of the desktop image among users in in a synchronized way is very important, especially when a user wants to show input parameters of an application or wants to teach how to make an operation to the other user.

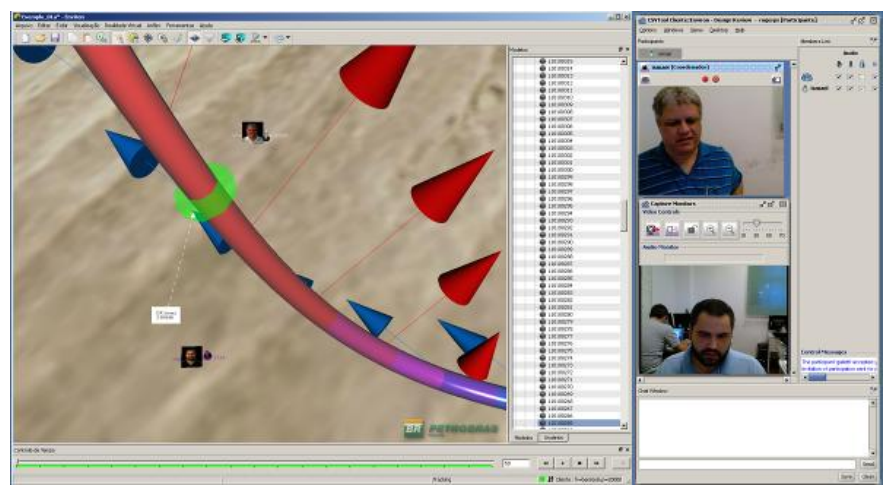

Fig.10. Closer look at an element of the riser.

Among other resources, it is possible to playback the simulation, examine pipes, sea waves and ship movements, and track elements in the risers that are subjected to extreme conditions (e.g., high stress values). It is also possible to select any element in a riser and examine it carefully; especially those elements in places subjected to great stress, such as the joints connection and the TDP (Touch Down Point, the first point of the riser that touches the seabed).

At the end of the session both users will have all the information exchanged during the collaborative session (annotations, measurements, etc) in their local copy. This information represents the state of the collaborative visualization session and can be stored in a file in a file that can be loaded again in the future toreconstruct the scenario that was former analyzed.

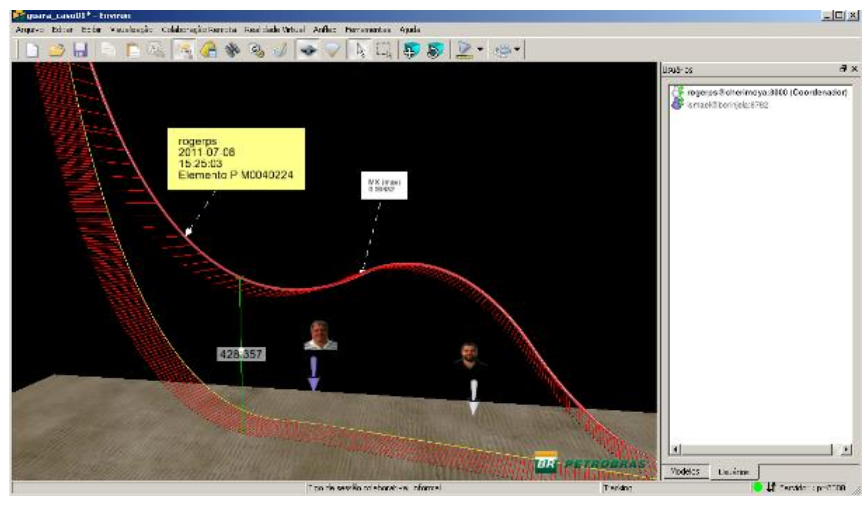

Fig.11. Users in a collaborative visualization session.

Fig.11 shows another collaborative visualization session, with another case study. The picture shows a 3D annotation created automatically, and another annotation created by one of the users, commenting about an element in the upper riser.

As can be seen, in the users tab the awareness mechanism shows information about the status of the user (online, offline) and his/her role in the session (coordinator or participant).

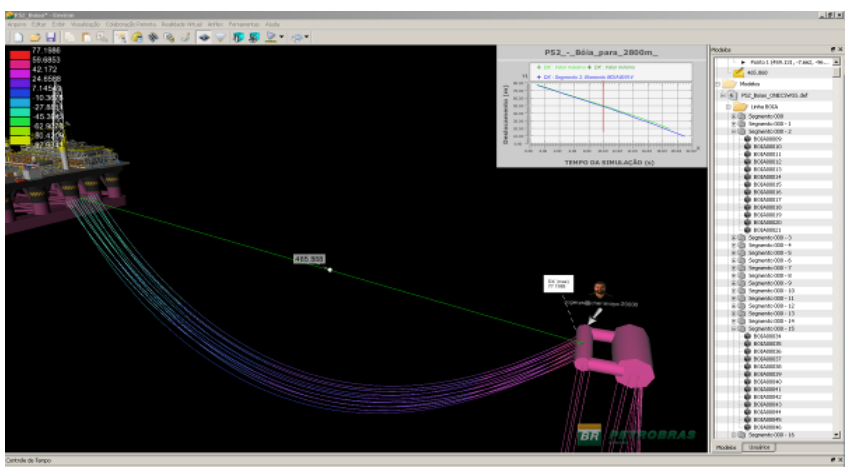

Fig.12. Users monitoring the behavior of marine buoyant.

Fig.12 shows an engineering project where the users want to study the movement of a buoyant, used to reduce the stress on the risers. Using a buoyant, the movement of the platform hull can be decoupled from the movement of the riser system. In this example users are monitoring the distance between the buoyant and the platform, while observing the behavior of some force on the risers.

\subsection{Design Review Workflow}

Design review is the process of checking the correctness and consistency of an engineering project while making the necessary adjustments. CEE is very helpful in this process, for instance allowing evaluating the safeness of different emergency escape pathways in case of an emergency.

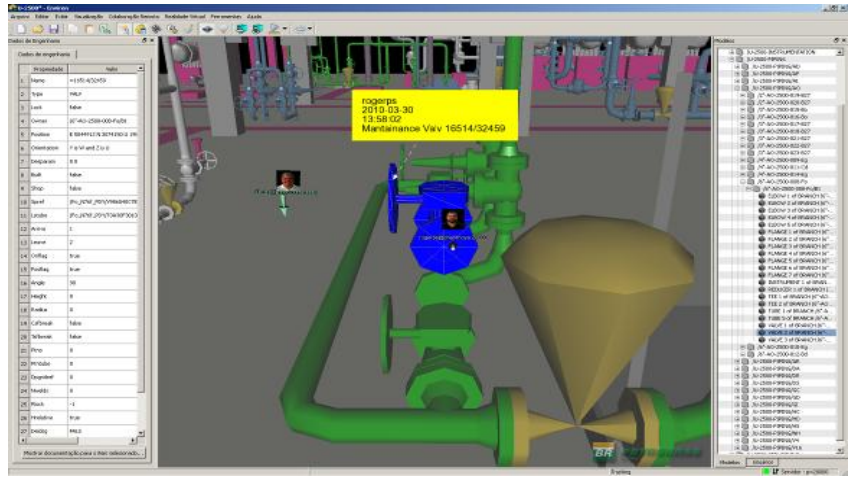

Fig.13. Maintenance plan enriched with annotations.

The design review workflow is a simplified version of the riser analysis workflow, where the workflow engine invokes the server to create a collaborative visualization session with the support of videoconference. In the session, users manipulate engineering objects, create $3 \mathrm{D}$ annotations and make 3D measurements in the model.

The ability to move, rotate and scale objects is important for various purposes, such as joining models, viewing hidden areas, planning the placement of new devices, and simulating a maintenance or intervention operation in a process plant. Moreover, integration with an engineering database from the CAD system is useful to create annotations emphasizing critical parts (Fig.13). Comments attached to objects can also be used as recommendations for project management.

Fig.14 shows a measurement taken for planning the 
movement of a large tank. Users create annotations to guide the maintenance procedure and animate the entire operation.

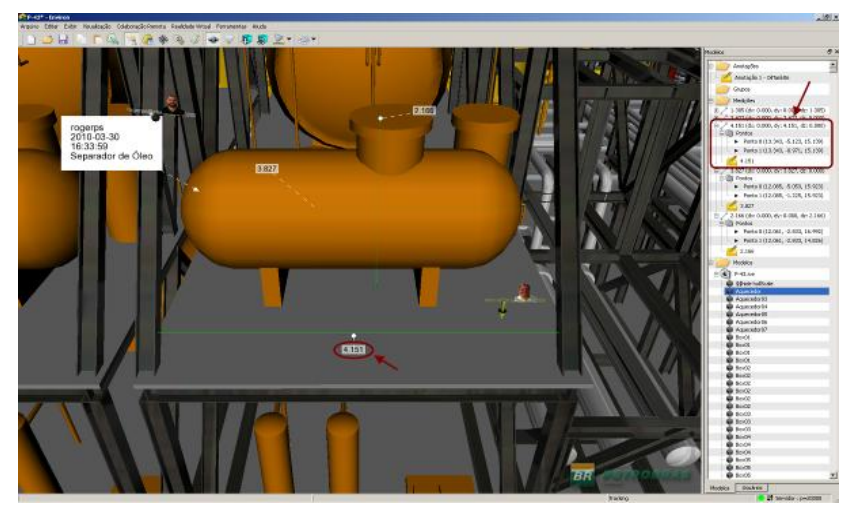

Fig.14. 3D measurements in a CAD model.

\subsection{Virtual Guided Tour}

In a Virtual Guided tour a user can follow the movements of another user, sharing the same view of the model. This modality of interaction is implemented creating a Classroom collaborative session, where the coordinator of the session creates virtual paths through critical spots in the scene in order to demonstrate or discuss possible anomalies in the results of the simulation with other users.

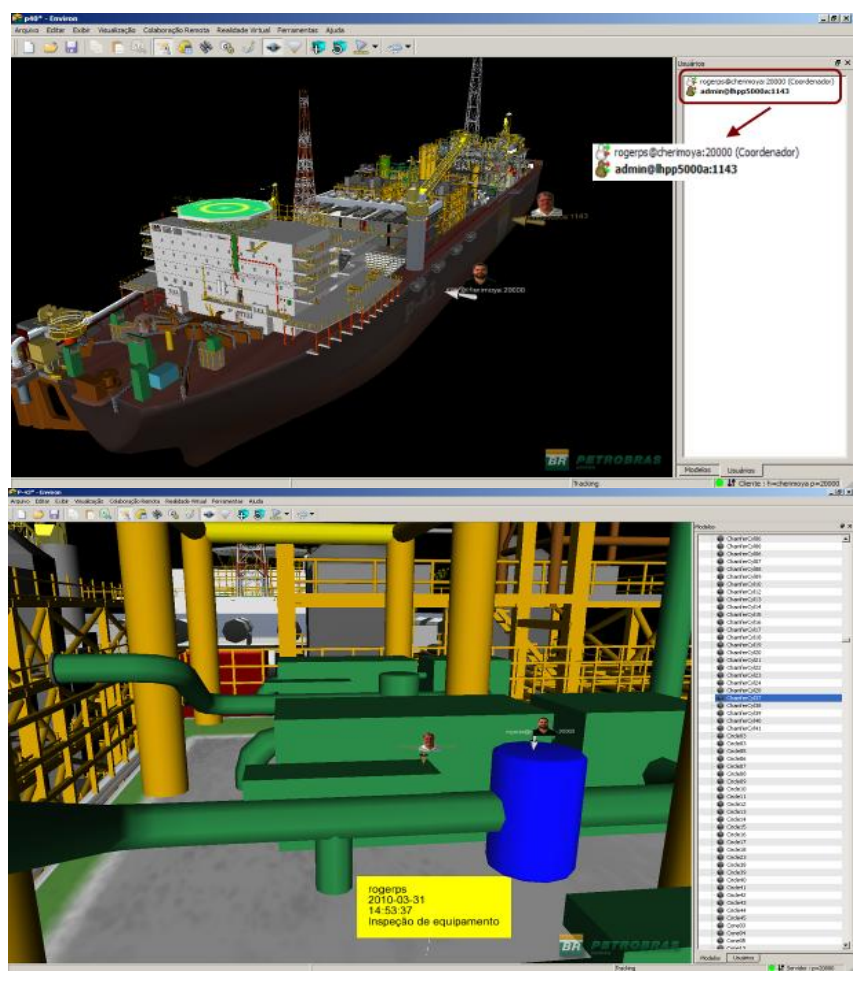

Fig.15. Virtual guided tour (Color Plate 4).

When following these paths the camera movements of the coordinator are retransmitted to other participants in the session allowing them to share the same view. This is also a very valuable tool for improving the knowledge of the simulation.

Fig.15 shows a collaborative visualization session, with both users following a 3D path passing through important points on an oil platform (Fig.15a) and in Fig.15b an annotation is created to some information associated to the selected object (in blue) on the platform. This way a maintenance operation can be defined to train new users or a commission of new equipment can be programmed and visualized.

Observe in the Fig.15 that the Awareness mechanism shows the icons of each user with 2 green lights, one for input and another for output. When changing to a Classroom or Lecture collaborative session the state of the coordinator and all participants change accordingly and also its representative icons on the users tab in the VRV GUI. The coordinator change its state to SendOnly (upper arrow green, lower arrow red) while each participant change its state to ReceiveOnly (upper arrow red, lower arrow green).

\section{CONCLUSIONS}

A collaborative environment was developed to improve the execution of large-scale engineering projects, such as offshore engineering projects. With the integration of VR technologies into the workflows used by team members, we expect to improve the use of VR in offshore engineering projects, as illustrated in

Fig.16.

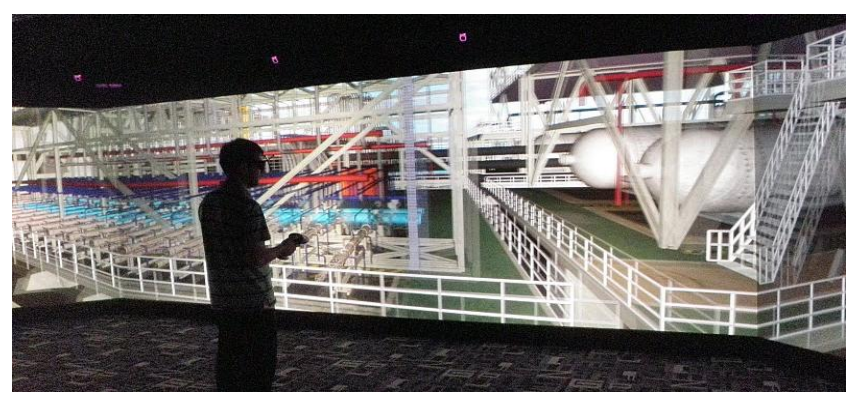

Fig.16. User in a cave during VR Collaboration.

It is clear that better visualization resources and techniques improve the quality of engineering projects, but users, in general, do not want to spend much time preparing their content to be viewed in a complex immersive multi-projection environments. The CEE simplifies the daily job of engineers, once they are able to collaboratively visualize their simulation results both on a desktop and on an immersive system, without having to change anything in their models. CEE also allows the users transparently execute their simulations as scientific workflows on a grid and visualize their results afterwards.

A specialized web interface was created where users can start immersive or desktop collaborative sessions to accomplish their job.

From the offshore engineering point of view, the creation of customized scientific workflows to be used by the users during the project lifecycle in a structured and integrated way, constituting an effective collaborative problem-solving environment, is an important contribution.

From the VR and Visualization point of view, CEE approach treats them as first class tools, exploring their potential for 
facilitating information exchange and common understanding of complex problems.

In the future, it is estimated that many other organizations are going to start to use scientific workflows, which will become a common solution in highly complex enterprises where several areas must be integrated. Although this work is focused on a solution for offshore engineering projects, we believe that the system could also be used in other areas.

Other future work is the integration of better avatars for VR collaboration, although 2D icons of people is a simple alternative, the integration of real-time 3D avatars of a reconstructed person will improve the benefits of the virtual simulation[36].

\section{REFERENCES}

[1] E. Tufte. The Visual Display of Quantitative Information. In Graphics Press, Cheshire, Connecticut.1983.

[2] E. J. Dodd. Visualization and Collaboration for the On Demand Upstream Petroleum Enterprise. IBM, Toronto, CA.2004

[3] E. N.Houstis, E. Gallopoulos, R. Bramley and J. R. Rice. Problem-Solving Environments for Computational Science, in IEEE Comput. Science and Engineerin, 4(3),18-21.1997.

[4] I. H. F. Santos, A. B. Raposo, M. Gattass. Software Architecture for an Engineering Collaborative Problem Solving Environment, in IEEE Software Engineering Workshop, SEW 2008, p.43-51, Greece, 2008

[5] E. Deelman, D. Gannon, M. Shields andI. Taylor. Workflows and e-Science: An overview of workflow system features and capabilities, Future GenerationComputer Systems, 25(5), p. 528-540. 2009.

[6] C. T. Pozzer, A. B. Raposo, I. H. F. Santos, J. L. E. Campos, L. P. Reis. CSVTool - A Tool for Video-Based Collaboration, in Proc. IX Simpósio Brasileiro de Sistemas Multimídia e Web - WebMedia, 353-367. 2003.

[7] OASIS Web Services Business Process Execution Language (WSBPEL)www.oasis-open.org/committees/tc_home.php? wg_abbrev=wsbpel

[8] A. B. Raposo, E. T. L. Corseuil, G. N. Wagner, I. H. F. Santos and M. Gattass. Towards the Use of CAD Models in VR Applications. In Proc. ACM SIGGRAPH Conf. VR-Continuum and its Applications in Industry, VRCIA, 67-74.2006.

[9] V. Mann and M. Parashar. DISCOVER: a computational collaboratory for interac-tive grid applications, in Grid computing: making the global infrastructure a reality, F. Berman, G. Fox, T. Hey (eds). Wiley: New York, pp. 727-744.2003.

[10] A. Paventhan, K. Takeda, S. J. Cox and D.A. Nicole. Leveraging Windows Workflow Foundation for Scientific Workflows in Wind Tunnel Applications, in Proceedings of the 22nd International Conference on Data Engineering Workshops, ICDEW'06, Atlanta, Georgia, April 2006.

[11] S. P.Callahan, J. Freire, E. Santos, C. E. Scheidegger, C. T. Silva and H. T Vo. VisTrails: Visualization meets Data Management, in Proc. ACM SIGMOD International Conference on Management of Data, 745-747. 2006.

[12] Eclipse BPEL Project. www.eclipse.org/bpel/

[13] S. G. Parker, M. Miller,C. D. Hansen, andC. R. Johnson. An integrated problem solving environment: The SCIRun computational steering system, in Proc. 31st Hawaii Int. Conf. System Sciences, HICSS-31, 147-156.1998.

[14] Paraview website. 2011. http://www.paraview.org

[15] O. Kreylos, G. Bawden, T. Bernardin, M. I. Billen, E. S. Cowgill, R. D. Gold, B. Hamann, M. Jadamec, L. H. Kellogg, O. G. Staadt and D. Y. Sumner. Enabling Scientific Workflows in Virtual Reality, in Proc. ACM SIGGRAPH Virtual-Reality Continuum and its Applications in Industry, VRCIA, 155-162.2006.

[16] R. High, S. Kindler andS. Graham. IBM SOA Foundation: An architectural introduction and overview, IBM developer Works, Nov/2005.

[17] R. Soma, A. Bakshi, A. Orangi, V. K. Prasanna and W. D. Sie. Service-Oriented Data Composition Architecture for Integrated Asset Management. SPE Intelligent Energy Conference and Exhibition (IECE).2006.
[18] H. Klie, W. Bangerth, M. Wheler, M. Parashar andV. Matossian. Parallel well location optimization using stochastic algorithms on the Grid computational framework, in Proc. 9th European Conference on the Mathematics of Oil Recovery (ECMOR IX).2004.

[19] K. Gruchalla. Immersive well-path editing: investigating the added value of immersion, in Proc. IEEE VR, VR04, 157-164, Chicago, USA.2004.

[20] C. Cruz-Neira, D. J. Sandin, T. A. DeFanti, R. V. Kenyon andJ. C. Hart. The Cave: audio visual experience automatic virtual environment, Communications of ACM, 35-6.1992.

[21] VRGeo Consortium Project. 2011. http://www.vrgeo.org

[22] A. Simonand S. Scholz. Multi-Viewpoint Images for Multi-User Interaction, in Proc IEEE Virtual Reality Conference, VR '05, pp. 107-113, March 2005.

[23] A. Simon. First-person experience and usability of co-located interaction in a projection-based virtual environment, in Proceedings of the ACM Symposium on Virtual Reality Software and Technology, VRST'05, Monterey, November 7-9, 23-30, CA, USA.2005.

[24] Y. L. Simmhan, B. Plale, D. Gannon.A survey of data provenance in e-science, in SIGMOD Record, vol. 34(3), pp 31-36, 2005.

[25] W. Emmerich, B. Butchart, L. Chen, B. Wassermann, S. L. Price. Grid Service Orchestration Using the Business Process Execution Language (BPEL), in Journal of Grid Computing v3(283-304), Springer 2006

[26] W. Lee, S. McGough, S. Newhouse and J. Darlington. A standard based approach to job submission through web services, in Proc. of the UK e-Science All Hands Meeting, pp. 901-905. UK EPSRC.2004

[27] E. C. Mourelle, E. C. Gonzalez and B. P. Jacob. ANFLEX Computational System for Flexible and Rigid Riser Analysis, in Proceedings of the 9th International Symposium on Offshore Engineering.1995.

[28] C.A.Ellis. Workflow technology. In Computer Supported Co-operative Work, M. Beaudouin-Lafon Ed., vol. 7 of Trends in Soft Series, John Wiley\&Sons, pp29-54, Chichester.1999

[29] M. Weske, M. Vossen, G. Medeiros andC. B. Pires, Workflow Management in Geoprocessing Apps. Tech. Report No.04-98-I, Univ.Muenster, Germany.1998.

[30] I. H. F. Santos, V. Braganholo, M. Mattoso, B. P. Jacob and C. Albrecht. Integrating The Galileo Applications For Simulation of Offshore Systems Via The GXML Unified Format. XXX CILAMCE - Iberian Latin American Congress on Computational Methods in Engineering. Buzios, Brazil.2009.

[31] H. Fuks, A. Raposo, M. A. Gerosa, M. Pimentel and C. J. P. Lucena. The 3C Collaboration Model,in Encyclopedia of E-Collaboration, Ned Kock (org), Information Science Reference, pp. 637-644, New York.2008.

[32] HDF Group. HDF5 File Format Specification Version 2.0. Available athttp://www.hdfgroup.org/HDF5/doc/H5.format.html

[33] A. B. Raposo, I. H. F. Santos, L. P. Soares, G. Wagner, E. Corseuil andM. Gattass. Environ: Integrating VR and CAD in Engineering Projects. IEEE Computer Graphics \& Applications, 29(6), p.91-95.2009.

[34] C. Ware, C. Gobrecht and M. Paton. Dynamic adjustment of stereo display parameters. In IEEE Transactions on Systems, Man and Cybernetics, vol. 28, pp. 56-65.1998.

[35] S. F. Senra, F. N. Correa, B. P. Jacob, M. M. Mourelle and I. Q. Masetti., Towards the integration of analysis and design of mooring systems and risers, part I: Studies on a semisubmersible platform, in $O M A E 2002$, v. 28046. 2002.

[36] L. P.Soares, C. Ménier, B. Raffin and J. Roch.. Work Stealing for Time-constrained Octree Exploration Application to Real-time 3D Modeling, in Proceedings of the Seventh Eurographics Workshop on Parallel Graphics \& Visualisation, presented at VII Eurographics Symposium on Parallel Graphics and Visualization. 2007.

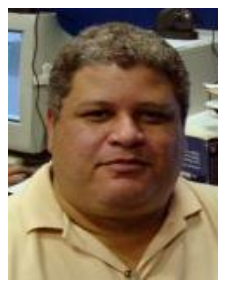

Ismael H. F. dos Santos works for Petrobras (Brazilian Oil company) since 1987. He holds a PhD in Computer Graphics, specialized in Virtual Reality, from Pontificial Catholic University of Rio de Janeiro and collaborates with the Computer Graphics Technolgy Group - TecGraf since 2000. He also has a master's degree in Applied Mathematics from the Federal University of Rio de Janeiro and he also lectures in many software development courses both internal and external to

Petrobras. 


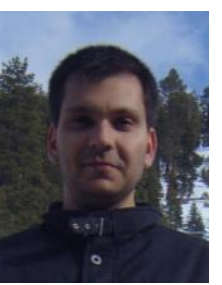

Luciano P. Soares holds a $\mathrm{PhD}$ in Electrical Engineering from Polytechnic School, University of São Paulo in Brazil. He was a postdoctoral research at the Instituto Superior Técnico in Portugal, Institut National de Recherche en Informatique et en Automatique in France and Instituto Superior de Ciências do Trabalho e da Empresa in Portugal. His research interests include, immersive multi-projection systems, real-time 3D computer graphics and high performance computing. He is currently a researcher at the Tecgraf, Computer Graphics Technology Group in PUC-Rio working in several projects with Petrobras.

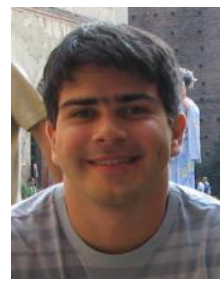

Felipe Carvalho graduated in Data Processing from the Federal University of Amazonas (2001), Master in Computer Science from the Pontifical Catholic University of Rio de Janeiro (2004) and $\mathrm{PhD}$ in Computer Science from the Pontifical Catholic University of Rio de Janeiro (2009). He is currently a researcher at the Computer Graphics Group - Tecgraf the Catholic University of Rio de Janeiro. He has experience in computer science, with emphasis on 3D visualization, 3D Interaction, Virtual Reality, Augmented Reality, Development of Non-conventional devices and computer vision.

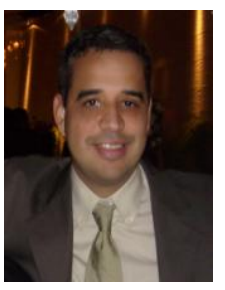

Alberto Barbosa is Assistant Professor at the Dept. of Informatics / PUC-Rio, project coordinator at Tecgraf/PUC-Rio and FAPERJ researcher. DSc in Electrical/Computer Engineering at the State University of Campinas, Brazil. Current interests: Virtual Reality, 3D interaction, groupware, $\mathrm{HCI}$, and computer graphics, with more than 120 publications in these areas. Projects supported by: Petrobras, CNPq, FINEP, FAPERJ and RNP. Distinguished young scholar, PUC-Rio and NVIDIA Academic Partner. 